\title{
Relación entre capacidad aeróbica y variables antropométricas en mujeres jóvenes físicamente inactivas de la ciudad de Concepción, Chile
}

\author{
Relationship between aerobic \\ capacity and antrophometric variables \\ in young women physicaly inactivite \\ from Concepción city, Chile
}

\begin{abstract}
Purpose: to determine which anthropometric variable (body mass index (BMI), waist circumference (WC) or waist-to-height ratio $(W / H t)$ has a better relationship with VO2peak in young physically inactive women from Concepción, Chile. Methods: 31 physically inactive women from Concepción, Chile (age $20.39 \pm 1.58$ years; weight $59.20 \pm 7.84 \mathrm{Kg}$; height $1.60 \pm 0.05 \mathrm{~m}$ ) were assessed in three stages: general information collection, anthropometric measuring (weight, height, WC, W/Ht, and BMI), and aerobic capacity determination, using Bruce's protocol associated to ergospirometry. For statistical analysis, Pearson's correlation coefficient was used, with $p<0.05$. Results: there is an inverse and moderate relationship between $B M I, W C$, and $W / H t$ with VO2peak in young physically inactive women from Concepción. Chile. W/Ht shows a higher correlation ( $r=-0.57$; $p<0.01)$ than CC $(r=-0.49 ; p<0.01)$ and IMC $(r=-0.45 ; p=0.01)$. Conclusions: W/Ht shows a better relationship with VO2peak than WC and BMI in young physically inactive women from Concepción, resulting in the anthropometric variable that most precisely determines cardiometabolic risk.
\end{abstract}

Key words: BMI; waist-to-height ratio; waist circumference; $\mathrm{VO}_{2}$ peak; cardiometabolic risk.
Andrea González R. (1)

Óscar Achiardi T. (2)

(1) Escuela de Kinesiología, Laboratorio de Ciencias Aplicadas a la Actividad Física, Pontificia Universidad Católica de Valparaíso, Valparaíso, Chile. (2) Escuela de Kinesiología, Laboratorio de Análisis del Movimiento Humano, Pontificia Universidad Católica de Valparaíso, Valparaíso, Chile.

Dirigir la correspondencia a: Andrea González Rojas Avenida Universidad $\# 330$,

Curauma, Valparaíso, Chile Teléfono (56) (032) 2274046 E-mail: andrea.gonzalez.r@ucv.cl

Este trabajo fue recibido el 3 de Julio de 2015 y aceptado para ser publicado el 30 de Octubre de 2015.

que niveles altos de capacidad aeróbica se asocian a un perfil metabólico favorable en adolecentes hispanos con sobrepeso y con normopeso (5). En contraparte, una baja capacidad aeróbica es un potente predictor de eventos cardiovasculares, morbilidad y mortalidad temprana en la edad adulta $(2,6)$.

Una de las principales amenazas para la reducción de la capacidad aeróbica es la inactividad física. Ésta constituye un grave problema de salud pública a nivel mundial y ha aumentado con el tiempo, considerándose "el problema de salud pública más grande del siglo XXI y uno de los factores de riesgo de enfermedades crónicas más prevalentes" (7). En Chile, tal como se demostró en la última Encuesta Nacional de Salud (ENS) del año 2010, la inactividad física alcanza 88,6\% de la población, siendo mayor en las mujeres $(92,9 \%)$ que en los varones (84\%), consolidándose como uno de los principales problemas de salud y factor de riesgo cardiometabólico (RCM) 
en la población (8).

Inactividad física se define como "el no cumplimiento de cualquiera de 3 criterios: realizar 30 minutos de actividad física de intensidad moderada al menos 5 días por semana, 20 minutos de actividad física vigorosa al menos 3 veces por semana o una combinación que permita lograr la utilización de 600 equivalentes metabólicos (METs) por semana" (9). Diversa evidencia sugiere que el tiempo que una persona pasa sentada, tiene efectos adversos sobre la salud cardiovascular y metabólica, acompañándose por una mayor predisposición a la disfunción, atrofia progresiva y pérdida de capacidad física (10). La inactividad física aumenta el riesgo relativo de padecer al menos 35 condiciones patológicas y es un factor de riesgo de muerte prematura y enfermedades crónicas (6). Mantenerse viendo televisión por tiempo prolongado se asocia con un mayor riesgo de incidencia de diabetes tipo 2 (DM2), enfermedad cardiovascular, obesidad y diversas causas de muerte (11).

De la mano con la inactividad física, el sobrepeso y la obesidad son otras de las condiciones que pueden afectar negativamente la capacidad aeróbica, siendo otro de los graves problemas de salud pública a nivel mundial (3). En Chile, la última encuesta nacional de salud (ENS) del año 2010, el porcentaje de personas con sobrepeso y obesidad es $67 \%$ (8.900.000 personas), $6 \%$ más que en la misma encuesta aplicada el año 2003 (61\%, es decir, aproximadamente 6.800 .000 personas). Al diferenciar por sexo, 30,7\% de las mujeres y $19,2 \%$ de los varones presentan obesidad, siendo este porcentaje mayor en las mujeres respecto a los varones, independiente del rango etario, lo que evidencia que el género femenino se encuentra en un mayor riesgo de sufrir eventos cardiometabólicos (8).

Entre las características de los sujetos obesos o con sobrepeso, la acumulación de adiposidad, sobre todo visceral abdominal, ha demostrado estar fuertemente asociada con un conjunto de anormalidades metabólicas y contribuye a la aparición de resistencia a la insulina, síndrome metabólico, enfermedades cardiovasculares y DM2 (3).

La utilización de métodos antropométricos en el diagnóstico y valoración clínica del sobrepeso y la obesidad, es especialmente relevante por ser relativamente simples, no invasivos, baratos, y no exigir alto grado de habilidad técnica y entrenamiento para ser aplicados. Además, teniendo en cuenta su eficacia, son una alternativa bastante utilizada en estudios poblacionales sobre obesidad y distribución regional de la grasa. Dentro de las herramientas clínicas de mayor utilización se encuentra el índice de masa corporal (IMC), la circunferencia de cintura (CC) y más recientemente el índice cintura-talla (ICT), que relacionan el peso o el perímetro abdominal con diferentes segmentos del cuerpo y presentan correlaciones altas y positivas entre sí (4). De ahí que su alteración tenga un valor particular para el diagnóstico de sobrepeso y obesidad, y como factor de riesgo y pronóstico de aparición de complicaciones secundarias (12).

EI IMC es un índice que permite diagnosticar el estado nutricional de un sujeto dividiendo su peso (en $\mathrm{kg}$ ) por su estatura (en mt) al cuadrado. Es de fácil utilización clínica, pero tiene como limitante que no distingue la masa magra de la masa grasa, por tanto no representa fielmente la distribución de la grasa en el organismo, por lo que algunos individuos que presentan peso normal o sobrepeso leve (según IMC) con una distribución anormal de la grasa corporal, podrían estar en alto riesgo de padecer por ejemplo DM $2(4,13)$. Aunque el IMC es de uso común y fácil de calcular, la evidencia científica disponible no ha sido capaz de aclarar su potencia como predictor de RCM, en comparación con otras medidas antropométricas $(4,13)$.

La CC es capaz de valorar la acumulación central de la grasa y es la medición más simple y de probada utilidad con estos fines. Sin embargo, al tratarse de una medida absoluta, no tiene en cuenta la influencia que puede sufrir por las dimensiones corporales de cada individuo. Es decir, se valora con el mismo criterio la circunferencia abdominal de una persona de 1,5 m de estatura, que otra con más de $2 \mathrm{~m}$. Esta dificultad se ha tratado de subsanar con el ICT, que intenta situar la circunferencia abdominal en función de la estatura y es calculado dividiendo la CC de una persona, por su estatura. Su punto de corte parece moverse alrededor de 0,5 es decir, la CC no debiera sobrepasar la mitad de la talla para mantener un bajo RCM (12). Diversas investigaciones han mostrado su utilidad para predecir este riesgo, lo que explicaría por qué este índice está cobrando protagonismo, e incluso, quizás podría ser más adecuado que la CC y el IMC para valorarlo en diversas condiciones $(12,14)$. A pesar de lo anterior, existen resultados controversiales al respecto, ya que mientras algunos autores lo reconocen como el mejor predictor de riesgo cardiovascular en sujetos con síndrome metabólico (14 - 16), otros que han encontrado mejores resultados si se utiliza la CC en mujeres (17) o el índice cintura/cadera (18).

En base a lo anterior, no todas las mediciones antropométricas mencionadas han mostrado igual relación con el RCM. Considerando que uno de los componentes de la función cardiorrespiratoria es el VO2peak, pudiera ser que alguna de ellas tenga mejor relación con la dimensión de ese riesgo asociado a la capacidad aeróbica. Medidas antropométricas que se correlacionen mejor con la capacidad aeróbica, podrían servir como aproximación más precisa del estado de salud de un individuo (2), representando de forma específica su RCM cuando existe influencia tanto de obesidad y sobrepeso, como de una pobre capacidad aeróbica. A nuestro saber, el único estudio que ha valorado la relación entre estas variables antropométricas y la capacidad aeróbica fue realizado en una población de adolescentes, de ambos géneros, sin considerar su nivel de actividad física y utilizando una estimación indirecta del consumo de oxígeno, por lo que sus resultados difícilmente pueden ser extrapolados a la población en mayor riesgo, como son las mujeres físicamente inactivas (4).

Surge entonces la pregunta de investigación ¿cuál de las tres evaluaciones antropométricas mencionadas (IMC, CC e ICT), presenta mejor relación con la capacidad aeróbica manifestada como $\mathrm{VO}_{2}$ peak de mujeres inactivas físicamente? El objetivo de este estudio es determinar cuál de las variables antropométricas (IMC, CC e ICT), tienen mejor relación con el $\mathrm{VO}_{2}$ peak en mujeres jóvenes físicamente inactivas de la ciudad de Concepción, Chile.

\section{SUJETOS Y MÉTODOS}

Estudio de tipo transversal, correlacional, que cumplió con la Declaración de Helsinki y contó con la aprobación del Comité de Ética de la Universidad Católica de la Santísima Concepción (UCSC) para su ejecución.

\section{Participantes}

Se realizó el cálculo del tamaño muestral utilizando la fórmula de Levy P. y Lemeshow S., (19) para estimación de una media. Se utilizó como referencia la variable circunferencia de cintura (CC) que es aquella que presenta mayor variabilidad en la literatura, con una desviación estándar de $8,27 \mathrm{~cm}$ según 
Burns et al en 2013. Utilizando un nivel de confianza del 95\% y una precisión del 3\%, el tamaño muestral mínimo obtenido fue de 29 participantes.

31 mujeres estudiantes universitarias de la ciudad de Concepción, Chile, con una edad promedio de 20,39 $\pm 1,58$ años, un peso corporal de 59,20 $\pm 7,84 \mathrm{Kg}$ y una estatura de 1,60 \pm 0,05 mt fueron reclutadas como voluntarias para participar en este estudio. Los criterios de inclusión fueron: ser mujer estudiante universitaria, con edad de 18 a 25 años, ser físicamente inactiva, considerando inactividad física como "el no cumplimiento de alguno de estos 2 criterios: realizar 30 minutos de actividad física de intensidad moderada al menos 5 días por semana o 20 minutos de actividad física vigorosa al menos 3 veces por semana" (9). Como criterio de exclusión se consideraron: presentar alguna patología musculo-esquelética, neurológica, cardio-metabólica o respiratoria diagnosticada, esté o no en tratamiento, uso de medicamentos que pudieran intervenir el rendimiento físico (por ejemplo beta adrenérgicos, psicotrópicos, beta bloqueadores, fármacos que contengan pseudoefedrina o efedrina y cafeína, entre otros), obesidad tipo III (IMC $\geq 40 \mathrm{~kg} / \mathrm{m} 2$ (según la Organización Mundial de la salud) (20), debido al riesgo de someterse a un test de esfuerzo incremental maximal) y embarazo. Luego de recibir detallada información sobre el estudio, cada persona decidió si sería parte de él manifestando lo anterior mediante la firma de la carta de consentimiento informado.

\section{Evaluaciones clínicas}

Las participantes fueron evaluadas en tres etapas: en primer lugar se efectuó una entrevista para recolectar información general que permita caracterizar la muestra, en segundo lugar se determinaron los índices de adiposidad, evaluando el peso, la talla y circunferencia de cintura (CC) de acuerdo al protocolo recomendado por la International Society for the Advancement of Kinanthropometry (ISAK), descrito por Holway and Garavaglia (2009) (21). En base a esos datos se calculó el Índice cintura/Talla (ICT) (circunferencia de la cintura $(\mathrm{cm})$ /estatura $(\mathrm{cm})$ ) y el Índice de masa corporal (IMC) (peso en $\mathrm{kg} /(\text { estatura en } \mathrm{cm})^{2}$ ). En tercer y último lugar se determinó la capacidad aeróbica mediante un test incremental maximal en trotadora (protocolo de Bruce) asociado a ergoespirometría para cuantificar el consumo peak de oxígeno (VO2peak), normalizado por el peso.

\section{Análisis estadístico}

Los datos fueron expresados en promedios y desviaciones estándar. Para determinar la existencia de correlaciones entre el VO2peak y el IMC, ICT y CC, se utilizó el coeficiente de correlación de Pearson para cada grupo. El nivel de significancia estadística fue de $p<0,05$. Para los análisis estadísticos de utilizó el programa GraphPad Prism5.

\section{RESULTADOS}

Las características generales, antropométricas y $\mathrm{VO}_{2}$ peak de las participantes en el estudio se aprecian en la tabla 1. Las correlaciones encontradas entre las variables antropométricas (IMC-CC; IMC-ICT y CC-ICT) se muestran en la tabla 2 y las correlaciones encontradas entre las variables antropométricas y el VO2peak se aprecian en la tabla 3.

De acuerdo a los resultados mostrados en la tabla 2, existen correlaciones directas y altas entre el IMC y CC $(r=0,89 ; p \leq 0,01)$, IMC e ICT $(r=0,88 ; p \leq 0,01)$ y CC e ICT $(r=0,89 ; p \leq 0,01)$. En la tabla 3 , se aprecia que existen correlaciones inversas y moderadas entre el $\mathrm{VO}_{2}$ peak y las variables antropométricas estudiadas. Dicha relación fue mayor entre ICT y VO 2 peak $(r=-0,57 ; p \leq 0,01)$, seguido por

TABLA 1

Características generales, antropométricas y $\mathrm{VO}_{2}$ peak de las participantes del estudio $(\mathrm{n}=31)$.

\begin{tabular}{lr}
\hline Edad (años) & $20,39 \pm 1,58$ \\
Peso $(\mathrm{kg})$ & $59,20 \pm 7,84$ \\
Talla $(\mathrm{m})$ & $1,60 \pm 0,05$ \\
$\mathrm{CC}(\mathrm{cm})$ & $74,71 \pm 5,76$ \\
$\mathrm{IMC}(\mathrm{Kg} / \mathrm{m} 2)$ & $22,95 \pm 2,56$ \\
$\mathrm{ICT}$ & $0,46 \pm 0,03$ \\
$\mathrm{VO}_{2}$ peak $(\mathrm{ml} / \mathrm{kg} / \mathrm{min})$ & $33,93 \pm 5,07$ \\
\hline
\end{tabular}

Número de individuos entre paréntesis. Valores expresados en promedio \pm desviación estándar. CC: Circunferencia de cintura; ICT: índice cintura/talla; IMC: índice de masa corporal; VO2peak: consumo peak de oxígeno relativo al peso.

TABLA 2

Correlaciones de las variables antropométricas entre sí (IMC-CC; IMC-ICT y CC-ICT), de las participantes del estudio ( $\mathrm{n=31}$ ).

\begin{tabular}{lcc}
\hline & Correlación & $p$ \\
IMC-CC & 0,89 & $\leq 0,01 \mathrm{a}$ \\
IMC-ICT & 0,88 & $\leq 0,01 \mathrm{a}$ \\
CC-ICT & 0,89 & $\leq 0,01 \mathrm{a}$ \\
\hline
\end{tabular}

Número de individuos entre paréntesis. (a) Coeficiente de correlación de Pearson.

ICT: índice cintura/talla; IMC: índice de masa corporal; CC: circunferencia de cintura. 
la CC y VO2peak $(r=-0,49 ; p \leq 0,01)$ y finalmente el IMC con VO2peak $(r=-0,45 ; p=0,01)$.

\section{DISCUSIÓN}

Los resultados encontrados muestran que las relaciones entre las variables antropométricas evaluadas (IMC-CC; IMCICT y CC-ICT) son todas altas y significativas. Estos resultados son similares a los encontrados por Burns et al en 2013 y son esperables dado que el ICT se calcula en base a la CC, el IMC será mayor si el peso del sujeto es mayor, lo cual puede verse afectado directamente si el sujeto presenta una CC mayor y a su vez, el ICT se calcula en base a la talla, al igual que el IMC, estando ambos influenciados por la misma variable.

En cuanto a las variables antropométricas y el $\mathrm{VO}_{2}$ peak, si bien todas las variables antropométricas evaluadas (IMC, CC e ICT) presentan una relación inversa y moderada con el VO2peak en mujeres jóvenes físicamente inactivas, el ICT presenta una correlación mayor que la CC y ésta, a su vez, que el IMC. Esto indica que, entre las variables antropométricas estudiadas, el ICT es el que permite verificar con mayor precisión que la CC y que el IMC, el RCM asociado a la capacidad aeróbica de una mujer físicamente inactiva.

Estos resultados concuerdan con lo encontrado por Burns et al en 2013, quien evaluó la existencia de relaciones entre medidas antropométricas (IMC, CC, ICT y porcentaje de masa grasa) y rendimiento cardiorrespiratorio (mediante $\mathrm{VO}_{2}$ indirecto en el test de carrera de 1 milla y test de PACER) en estudiantes de ambos sexos que cursaban sexto, séptimo y octavo grado. Si bien sus resultados fueron similares en cuanto a que obtuvo correlaciones moderadas entre porcentaje de masa grasa e ICT con las pruebas de aptitud cardiorrespiratoria en ambos sexos, se debe cautelar el hecho de que su estudio fue efectuado en adolescentes y no en adultos. En cuanto al IMC, encontraron correlaciones débiles con los resultados de las pruebas de aptitud cardiorrespiratoria, sugiriendo que otras mediciones antropométricas como el ICT pueden ser indicadores más válidos que el IMC, de salud asociada a capacidad aeróbica (4). Dentro de las principales limitaciones del IMC se encuentran la incapacidad de distinguir entre masa grasa y masa libre de grasa y la distribución de la grasa corporal $(4,13)$, siendo una variable poco sensible, sobreestimando o subestimando la real condición antropométrica del sujeto. En cuanto a la CC, la principal limitación se basa en que no considera la estatura del sujeto, por lo cual dificulta hacer comparaciones entre individuos cuando se quiere identificar riesgo para la salud $(4,12)$.

Hernández y Duchi en 2015, platean en una revisión bibliográfica acerca del ICT y su utilidad para detectar riesgo cardiovascular y metabólico, que idealmente, la CC debe ser menos de la mitad de la talla, y que cuando dicho índice au- menta, es capaz de identificar personas que a pesar de tener IMC normal, pueden tener un riesgo metabólico elevado asociado con obesidad central (ICT mayor de 0,5), considerando que la acumulación excesiva de tejido adiposo visceral es uno de los más factores más influyentes en la aparición de un perfil cardiometabólico adverso y de síndrome metabólico, asociados al sobrepeso y la obesidad $(3,22)$. Si tenemos en cuenta que el ICT ha mostrado tener una elevada correlación con el porcentaje de masa grasa corporal, razón por la cual algunos profesionales lo reconocen como el mejor predictor de riesgo en pacientes con síndrome metabólico (SM) (equiparable al IMC en la predicción de DM2) (12), además de la necesidad de aumentar las investigaciones al respecto (23), este trabajo aporta fundamentos importantes enormemente al mostrar que esta variable antropométrica presenta una mejor correlación con la capacidad aeróbica (representada por el VO2peak) como una de las dimensiones del RCM.

EI ICT es una evaluación antropométrica fácil de realizar y con una relación mejor con el $\mathrm{VO}_{2}$ peak que la CC y el IMC, pero sobre la cual aún existen controversias y vacíos en la literatura. Por lo anterior, resulta de gran interés saber si presenta alguna relación con diversos marcadores de RCM en diferentes rangos etarios, sexos y etnias, para verificar si efectivamente puede ser considerado como un indicador clínico de ese riesgo, más preciso que el IMC y la CC. Al ser este el primer trabajo realizado en Chile que busca determinar cuál de las variables antropométricas evaluadas se relaciona mejor con el VO2peak, puede ser el inicio de una línea de investigación que aporte en la validación de herramientas clínicas de fácil y extensa utilización.

Dentro de las limitaciones del estudio se encuentra el hecho de que si bien el número de participantes incluidos sobrepasa el número mínimo calculado como muestra, sería interesante evaluar si los resultados encontrados se mantienen al realizar el estudio con un mayor número de participantes.

Por otra parte, dentro de las proyecciones de este estudio se encuentran evaluar el comportamiento de los varones bajo las mismas condiciones e incluir otras mediciones antropométricas, como por ejemplo el índice cintura cadera (ICC) y la circunferencia de cuello, con el objetivo de evidenciar su relación con el $\mathrm{VO}_{2}$ peak y así continuar la búsqueda de variables de aplicación clínica que puedan ayudar en la determinación del RCM asociado a la capacidad aeróbica de forma más precisa. Por otra parte, sería interesante evaluar el comportamiento de estas variables en diferentes rangos etarios y según diferentes niveles de actividad física, dado que tanto la edad como el nivel de actividad física de una persona pueden modificar tanto la capacidad aeróbica como la composición corporal, afectando las variables antropométricas y la capacidad aeróbica y por ende el RCM de la población.

\section{TABLA 3}

Correlaciones entre cada variable antropométrica y el VO2peak de las participantes del estudio $(n=31)$.

\begin{tabular}{lcc}
\hline & Correlación & $p$ \\
$V_{2}$ peak-ICT & $-0,57$ & $\leq 0,01 \mathrm{a}$ \\
$\mathrm{VO}_{2}$ peak-CC & $-0,49$ & $\leq 0,01 \mathrm{a}$ \\
$\mathrm{VO}_{2}$ peak-IMC & $-0,45$ & $0,01 \mathrm{a}$
\end{tabular}

Número de individuos entre paréntesis. (a) Coeficiente de correlación de Pearson.

ICT: índice cintura/talla; IMC: índice de masa corporal; CC: circunferencia de cintura; VO2peak: consumo peak de oxígeno relativo al peso. 


\section{CONCLUSIÓN}

En mujeres jóvenes físicamente inactivas de la ciudad de Concepción, Chile, existe una relación inversa y moderada entre el $\mathrm{VO}_{2}$ peak y el ICT, CC e IMC respectivamente. En orden decreciente, la relación es mayor entre el $\mathrm{VO}_{2}$ peak e ICT, luego entre $\mathrm{VO}_{2}$ peak y CC y finalmente entre $\mathrm{VO}_{2}$ peak e IMC.

\section{RESUMEN}

Objetivo: determinar cuál variable antropométrica (Índice de masa corporal (IMC), circunferencia de cintura (CC) o Índice cintura/talla (ICT) tiene mejor relación con VO2peak en mujeres jóvenes físicamente inactivas de la ciudad de Concepción, Chile. Sujetos y Métodos: 31 mujeres físicamente inactivas de la ciudad de Concepción, Chile (edad 20,39 $\pm 1,58$ años; peso $59,20 \pm 7,84 \mathrm{Kg}$ : talla 1,60 $\pm 0,05 \mathrm{~m}$ ) fueron evaluadas en tres etapas: recolección de información general, medición antropométrica (peso, talla, CC, ICT e IMC) y determinación de la capacidad aeróbica mediante el Protocolo de Bruce asociado a ergoespirometría. Para el análisis estadístico se utilizó el coeficiente de correlación de Pearson con $p<0,05$. Resultados: existió una relación inversa y moderada entre IMC, CC e ICT y VO $\mathrm{V}_{2}$ peak en mujeres jóvenes inactivas físicamente de la ciudad de Concepción, Chile. ICT presenta una correlación mayor $(r=-0,57 ; p<0,01)$ que $C C(r=-0,49 ; p<0,01)$ y que IMC $(r=-0,45 ; p=0,01)$. Conclusiones: ICT presenta mejor relación con el $\mathrm{VO}_{2}$ peak que la CC y que el IMC en mujeres jóvenes inactivas físicamente de la ciudad de Concepción, siendo la variable antropométrica que determina con mayor precisión el riesgo cardiometabólico.

Palabras clave: IMC; índice cintura/talla; circunferencia de cintura; $\mathrm{VO}_{2}$ peak; riesgo cardiometabólico.

Conflictos de interés: Los autores declaran no tener conflictos de interés.

Agradecimientos: Los autores desean expresar su agradecimiento al Laboratorio de Kinesiología Aplicada de la Universidad Católica de la Santísima Concepción (UCSC) por el apoyo brindado al facilitar el equipamiento para realizar este estudio y a los estudiantes de Kinesiología de la UCSC por su enorme ayuda durante el proceso de evaluación de las participantes.

\section{BIBLIOGRAFÍA}

1. Motl, R. \& Goldman, M. Physical inactivity, neurological disability, and cardiorespiratory fitness in multiple sclerosis. Acta Neurol Scand 2011; 123(2): 98-104. Doi:10.1111/ j.1600-0404.2010.01361.x.

2. Hulkkonen, J., Aatola, H., Pälve, K., Lehtimäki, T., HutriKähönen, N., Viikari, J., Raitakari, O. \& Kähönen, M. Determinants of exercise peak arterial blood pressure, circulatory power, and exercise cardiac power in a population based sample of Finnish male and female aged 30 to 47 years: the Cardiovascular Risk in Young Finns Study. BMC Cardiovasc Dis. 2014; 14: 35.

3. Kim, S., Kim, J-Y., Lee, D-C., Lee, H-S., Lee, J-W \& Jeon, J. Combined Impact of Cardiorespiratory Fitness and Visceral Adiposity on Metabolic Syndrome in Overweight and Obese Adults in Korea. PLoS ONE 2014; 9 (1): e85742. doi:10.1371/journal.pone.0085742

4. Burns, R., Hannon, J., Brusseau, T., Shultz, B. \& Eisenman, P. Indices of Abdominal Adiposity and Cardiorespiratory Fitness Test Performance in Middle-School Students. Obesity 2013; 2013: 1-8. doi: http://dx.doi. org/10.1155/2013/912460

5. Mesa, JL., Ortega, F., Ruiz et al. Anthropometric determinants of a clustering of lipid-related metabolic risk factors in overweight and non-overweight adolescents-influence of cardiorespiratory fitness: The AVENA study. Ann Nutr Metab 2006; 5 (6): 519-27. doi: 10.1159/000098144

6. Laye, M., Nielsen, M., Hansen, L., Knudsen, T. \& Pedersen. B. Physical Activity Enhances Metabolic Fitness Independently of Cardiorespiratory Fitness in Marathon Runners. Dis. Markers; 2015: p1-11 doi: http://dx.doi. org/10.1155/2015/806418

7. Wilson-Frederick, S., Thorpe, R., Bell, C.,Bleich, S., Ford, J. \& LaVeist, T. Examination of Race Disparities in Physical Inactivity among Adults of Similar Social Context. Ethn Dis 2014; 24(3): 363-9.

8. Encuesta Nacional de Salud 2010. Ministerio de Salud de Chile (MINSAL). Consultada en Junio de 2015. http://www.minsal.gob.cl/portal/url/item/bcb03d7bc28b64dfe040010165012d23.pdf

9. Hallal, C., Andersen, L., Bull, F., Guthold, R. \& Haskell, W. Global physical activity levels: surveillance progress, pitfalls, and prospects. Lancet 2012; 380: 247-57. Doi: http:// dx.doi.org/10.1016/S0140-6736(12)60646-1

10. González-Gross, M., Meléndez, A. Sedentarism, active lifestyle and sport: impact on health and obesity prevention. Nutr Hosp. 2013; 28 (Supl. 5): 89-98.

11. Kim, Y., Wilkens, L., Park, S-Y., Goodman, m., Monroe, $k$. and Kolonel, L. Association between various sedentary behaviours and all-cause, cardiovascular disease and cancer mortality: the Multiethnic Cohort Study. Internat J Epidemiol. 2013; 42: 1040-56. doi:10.1093/ije/dyt108.

12. Hernández, J. \& Duchi, P. Índice cintura/talla y su utilidad para detectar riesgo cardiovascular y metabólico. Rev Cubana Endocrinol. 2015; 26(1): 66-76.

13. Lamêgo, S., Perim, M., Geraldo, J. Association of WaistStature Ratio with Hypertension and Metabolic Syndrome: Population-Based Study. Arq Bras Cardiol. 2010; 95(2): 186-191.

14. Remón, I., González, O., Arpa, C. El índice cintura-talla como variable de acumulación de grasa para valorar riesgo cardiovascular. Rev Cubana Med Militar 2013; 42(3): 444-50.

15. Maffeis C, Banzato C, Talamini G. Waist-to-Height Ratio, a Useful Index to Identify High Metabolic Risk in Overweight Children. J Pediatr. 2008; 152: 207-13.

16. Moreira $M N$ ¿Qué medida antropométrica de exceso de peso discrimina mejor el riesgo cardiovascular? Med Clin (Barc). 2010; 134(9): 396-8.

17. Rodríguez MC, Cabrera A, Aguirre A, Domínguez S, Brito B, Almeida $D$, et al. El cociente perímetro abdominal/estatura como índice antropométrico de riesgo cardiovascular y de diabetes. Med Clin (Barc). 2010; 134(9): 386-91.

18. Cabrera Rode E, Bioti Y, Marichal S, Parlá J, Arranz C, Olano $R$, et al. Índice cintura-cadera contra perímetro cintura para el diagnóstico del síndrome metabólico en niños y adolescentes con familiares de primer grado diabéticos tipo 1. Rev Cubana Endocrinol. 2011; 22(3): 182-95.

19. Ruiz A. \& Morillo L. Epidemiología Clínica: Investigación clínica aplicada. Ed. Médica Panamericana, Bogotá, 2004.

20. Organización Mundial de la salud Consultada el 19 de Octubre de 2015. http://apps.who.int/bmi/index. jsp?introPage=intro_3.html

21. Holway, F. E., \& Garavaglia, R. Kinanthropometry of Group I rugby players in Buenos Aires, Argentina. Sports 
Sci. 2009; 27(11): 1211-1220. Doi: 10.1080/026404 10903207408.

22. Buchan, D., Young, J., Boddy, L., Malina, R. \& BakeR, J. Fitness and Adiposity Are Independently Associated with Cardiometabolic Risk in Youth. BioMed Res Internat. 2013;
2013: 1-6. Doi: http://dx.doi.org/10.1155/2013/261698

23. De Piero, A., Rodríguez, E., González, L. \& López, A. Sobrepeso y obesidad en un grupo de escolares españoles. Rev Chil Nutr. 2014; 41, (3): 264-71. Doi: http://dx.doi. org/10.4067/S0717-75182014000300006. 